\title{
CONSTRUCTION OF SINE AND COSINE HOLOGRAM OF BRAIN TUMOR IMAGE
}

\author{
Vinay Y. Jadhav ${ }^{1}$, Brintha Therese $\mathrm{A}^{2}$ \\ ${ }^{1}$ M.Tech, school of electronics engineering, VIT University, Tamilnadu, India \\ ${ }^{2} \mathrm{PhD}$, school of electronics engineering, VIT University, Tamilnadu, India
}

\begin{abstract}
Optical Scanning Holography is a technique in which an image is scanned by Time Dependent Fresnel Zone Plate and its intensity information is encoded into a two dimensional hologram. This technique not only records amplitude but also the phase of the object in case of various applications such as optical remote sensing, 3D encoding and decoding, 3D TV system. Optical scanning holography is an electronic process which is flexible, programmable and also has the capability of parallel processing. It has become a powerful technique over digital holography technique as it provides a very high processing speed, good quality color appearance, better sharpness and high resolution. An optical Fourier transform technique is used for its processing. Optical scanning holography is a real time system which involves the principle of optical heterodyne scanning. This paper proposes the construction of sine and cosine hologram of biomedical image such as brain tumor by using optical scanning setup. The holograms generated by this approach will be used for detection of tumor cells when these holograms are processed further in the reconstruction stage which will visualize more information of such a malignant cells. In the present work the construction stage of the hologram with optical set up has been accomplished and the simulation results of the hologram are observed by using MATLAB tool.
\end{abstract}

Key Words: - Holography, Heterodyne scanning, Fourier transform, Fresnel Zone Plate, Optical setup

\section{INTRODUCTION}

Brain tumor is inherently serious and life-threatening disease. Brain tumors include all tumors inside the cranium. They are created by an abnormal and uncontrolled cell division. So, for the detection of such malignant cells optical scanning holography technique is used as it provides better sharpness \& resolution which can visualize the infected tumor cells. Optical scanning holography is a novel concept in holographic optics. The success of creating holograph mainly depends on how the interference pattern is constructed and recorded. Biomedical imaging has been considered as the fastest developing field of biomedical systems. In case of biological specimens, analyzing the sectional intensity distribution is difficult. But by using optical scanning holography entire information of thick biological specimen is recorded into a $2 \mathrm{D}$ hologram by single lateral scanning. It aligns the cellular structures into a plane without overlapping [1]. Optical scanning holography $(\mathrm{OSH})$ has the lateral resolution better than $1 \mu \mathrm{m}$ with fluorescent specimens [2]. OSH has several applications such as holographic information transmission, 3D Coding and Decoding, 3D optical remote sensing, 3D microscopy and biomedical applications which requires real time processing [3]. Both on-axis and off-axis recording can be generated with OSH which further reduces resolution and channel bandwidth requirement in 3-D TV system [4]. Baoqun Zhao, Aili Qin, Huaying Wang have analyzed acquisition method for minute object using digital holography system [5]. Optical scanning holography has been investigated by different groups in the world such as Carter has investigated this approach for optical remote sensing applications [6]. The Xie's group implanted optical scanning technique for the applications of imaging through turbid media [7]. The homodyne detection has been investigated by Rosen et al. It shows that by using a linear arrangement of at least three holograms taken with three Fresnel Zone Plates with dissimilar phase values ,the twin image problem can be solved. However the phase shift generated in this technique introduces some error and uncertainty in the phase value of hologram [8].

In this paper we mainly focus on constructing the sine and cosine hologram of brain tumor image which can be used for further detection of malignant cells in the brain. The time dependent Fresnel zone plates used for $\mathrm{OSH}$ gives two on-axis holograms which does not produce a twin image after reconstruction [9]. In case of digital holography technique processing speed limits to several milliseconds, this limitation can be overcome by optical scanning technique as in case of optical scanning holography the processing speed is in several nanoseconds. Also it supports parallel processing approach which will be helpful in multi-tasking mechanism. Some other features like resolution, sharpness enhances the feasibility of this technique in most of the real time applications. 


\section{DESIGN METHODOLOGY}

It is proposed a new approach for diagnosis of brain tumor cells by using holographic findings. It is demonstrated as follows in Fig-1.

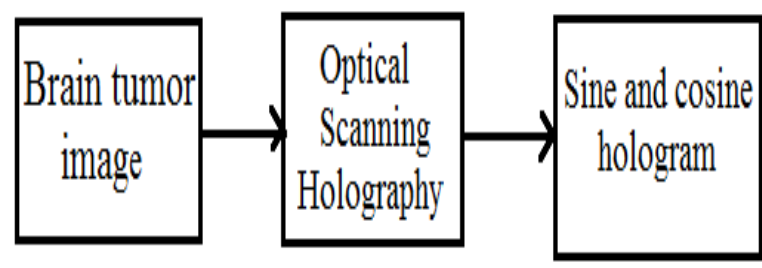

Fig -1 : Proposed Method

\section{OPTICAL SCANNING HOLOGRAPHY}

The Optical Scanning holography (OSH) is a technique which records holographic information of an object using heterodyne optical scanning investigated by T.C. Poon [3]. To get high quality hologram one should need to carefully arrange the optical elements and never change the position of any element during exposure. The scanning consists of two parts, namely recording and reconstruction stage. Fig-2 shows the experimental set up of optical scanning system for holographic recording. The Time Dependent Fresnel zone plate (TDFZP) is used for 2-D scanning of the object in the recording stage. The TDFZP is realized using a two-pupil heterodyne processing technique. Acousto-optic modulator (AOM) generates spherical wave of different frequency. The Fresnel Zone Plate is created by the superposition of a spherical wave of different frequencies and a plane wave. In this paper, 2D Sine Fresnel Zone Plate hologram of the brain tumor image is generated using time dependent Fresnel zone plate. The brain image is scanned and a heterodyne scanned current corresponding to malignant cells delivered as an output. This current contains FZP coded information of the image which mixed up to give demodulated signal. The demodulated signal is then synchronized with optical scanning system. And the information which is displayed is nothing but the 2D hologram or FZP coded information of brain tumor image. This holographic technique is commonly known as electronic holography.

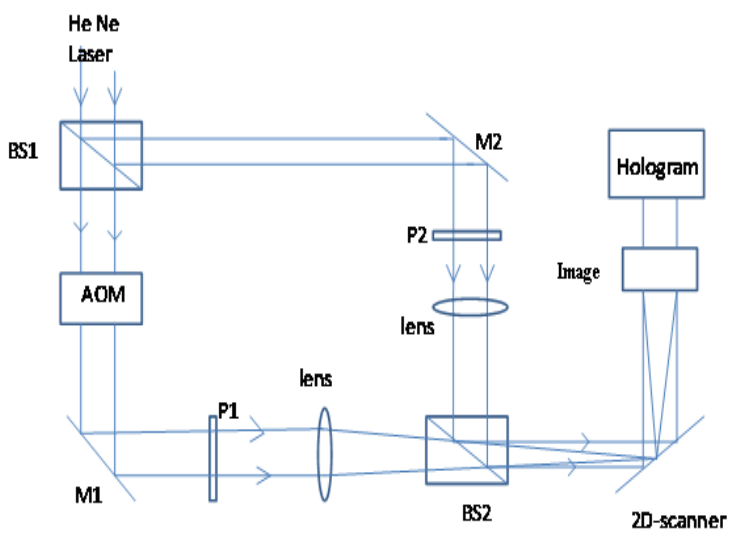

Fig-2 : Optical Setup for Holographic Recording
The He-Ne laser is used which is having an operating wavelength of $632 \mathrm{~nm}$ for stimulated emission of photons. The pupil function $\mathrm{p}_{2}(\mathrm{x}, \mathrm{y})$ is illuminated by a collimated laser at a temporal frequency of $\mathrm{w}_{0}$. The other pupil function $\mathrm{p}_{1}(\mathrm{x}, \mathrm{y})$ is illuminated by the laser of temporal frequency of $\mathrm{w}_{0}+\Omega$. These pupils are then combined by the beam splitter BS2 to focus the light onto the 2-D scanner which is located on the back focal plane of the two lenses. The combined optical beams are then used for 2-D scanning over an object which is located at a distance of D from the focal plane of the two lenses.

\section{MATHEMATICAL REPRESENTATION}

Consider a planar object located at $z=0$ and described by a transparency $T_{0}(x, y)$. The amplitude and phase of a light field at $z=0$ is given by complex function $\Phi(x, y ; z=0)$

Now Assuming plane wave illumination with unity amplitude and zero initial phase at $\mathrm{z}=0$

By Fresnel diffraction, the light field is given by,

$$
\Phi(x, y ; z)=\mathrm{T}_{0}(x, y) \otimes h(x, y ; z)
$$

Where, $h(x, y ; z)$ is free-space impulse response of the system. It is given by the following equation,

$$
h(x, y ; z)=\frac{i k_{0}}{2 \pi z} \exp \left[-i k_{0}\left(\frac{x^{2}+y^{2}}{2 z}\right)\right]
$$

Where, $k_{0}$ is the wave number of the light field which is given by, $k_{0}=\frac{2 \pi}{\lambda}$

The optical transfer function (OTF) of the heterodyne scanning system is given by,

$$
\mathrm{OTF}=\exp \left[-i k_{0}\left(\frac{k_{x}^{2}+k_{y}^{2}}{2 z}\right)\right]=H(x, y ; z)
$$

Where, $k_{x}$ and $k_{y}$ are spatial coefficients.

The equation obtained at the output of the scanner is given by,

$$
t(x, y)=\operatorname{Re}\left\{\left[\left|T_{0}\right|^{2} \otimes h(x, y ; z)\right]\right\}
$$


The two spatial domain output current equations are given by,

$$
t_{s}(x, y)=\operatorname{Re}\left[\int \mathrm{f}^{-1}\left\{\mathrm{f}\left\{\left|T_{0}(x, y ; z)\right|^{2}\right\} \mathrm{OTF}\right\} \mathrm{dz}\right]
$$

This equation can be rewritten in terms of convolution as,

$$
\begin{aligned}
& t_{s}(x, y)=\operatorname{Re}\left[\int\left\{\left|T_{0}\right|^{2} \otimes \mathrm{h}(\mathrm{x}, \mathrm{y} ; \mathrm{z})\right\} \mathrm{dz}\right] \\
& t_{c}(x, y)=\operatorname{Im}\left[\int \mathrm{f}^{-1}\left\{\mathrm{f}\left\{\left|T_{0}(x, y ; z)\right|^{2}\right\} \mathrm{OTF}\right\} \mathrm{dz}\right]
\end{aligned}
$$

It can be also written in convolution form as,

$$
t_{c}(x, y)=\operatorname{Im}\left[\int\left\{\left|T_{0}\right|^{2} \otimes \mathrm{h}(\mathrm{x}, \mathrm{y} ; \mathrm{z})\right\} \mathrm{dz}\right]
$$

Where, $t_{\mathrm{c}}(x, y)$ and $t_{\mathrm{s}}(x, y)$ represents the scanned and processed current information of $\left|T_{0}(\mathrm{x}, \mathrm{y} ; \mathrm{z})\right|^{2}$

From equation (2) the spatial domain equations becomes,

$$
\begin{aligned}
t_{s}(\mathrm{x}, \mathrm{y}) & =\int\left\{\left|T_{o}\right|^{2} \otimes\left(\frac{k_{0}}{2 \pi z}\right) \sin \left[\frac{k_{0}\left(x^{2}+y^{2}\right)}{2 z}\right]\right\} d Z \\
& =H_{\sin }(x, y) \\
t_{c}(x, y) & =\int\left\{\left|T_{0}\right|^{2} \otimes\left(\frac{k_{0}}{2 \pi z}\right) \cos \left[\frac{k_{0}\left(x^{2}+y^{2}\right)}{2 z}\right]\right\} d Z \\
& =H_{\cos }(x, y)
\end{aligned}
$$

Let, $\quad\left|T_{0}\right|^{2}=\delta(x, y) \delta\left(z-z_{0}\right)$ which is a point source located at $z_{0}$,

$$
\begin{aligned}
& H_{\text {sin }}(x, y)=\int\left\{\delta(x, y) \delta\left(z-z_{0}\right) \otimes\left(\frac{k_{0}}{2 \pi z}\right) \sin \left[\frac{k_{0}\left(x^{2}+y^{2}\right)}{2 z}\right]\right\} d Z \\
& H_{\text {sin }}(x, y)=\int\left\{\delta\left(z-z_{0}\right)\left(\frac{k_{0}}{2 \pi z}\right) \sin \left[\frac{k_{0}\left(x^{2}+y^{2}\right)}{2 z}\right]\right\} d Z
\end{aligned}
$$

After 2-D convolution involving $\mathrm{x} \& \mathrm{y}$ and integrating along $\mathrm{z}$, the above equations becomes,

$$
H_{\text {sin }}(x, y)=\left(\frac{k_{0}}{2 \pi z_{0}}\right) \sin \left[\frac{k_{0}\left(x^{2}+y^{2}\right)}{2 z_{0}}\right]
$$

$$
H_{\cos }(x, \mathrm{y})=\left(\frac{k_{0}}{2 \pi z_{0}}\right) \cos \left[\frac{k_{0}\left(x^{2}+y^{2}\right)}{2 z_{0}}\right]
$$

Equation (14) and (15) represents sine and cosine hologram respectively.

\section{SIMULATION RESULTS}

The simulations are performed by using MATLAB software. Brain tumor image is used as input in this technique for the simulation. The real and imaginary parts of the hologram are obtained in the simulation process with good resolution and sharpness. These holograms contain amplitude as well as phase information of the original image which will visualize more information regarding malignant cells of tumor. The amplitude of the source image can be used for the segmentation process which will extract the required information of cells after reconstruction stage. The simulation results are shown in following figures. Fig-3 shows the original brain tumor image. Fig-4 shows sine hologram of image and Fig-5 indicates cosine hologram of the tumor image.

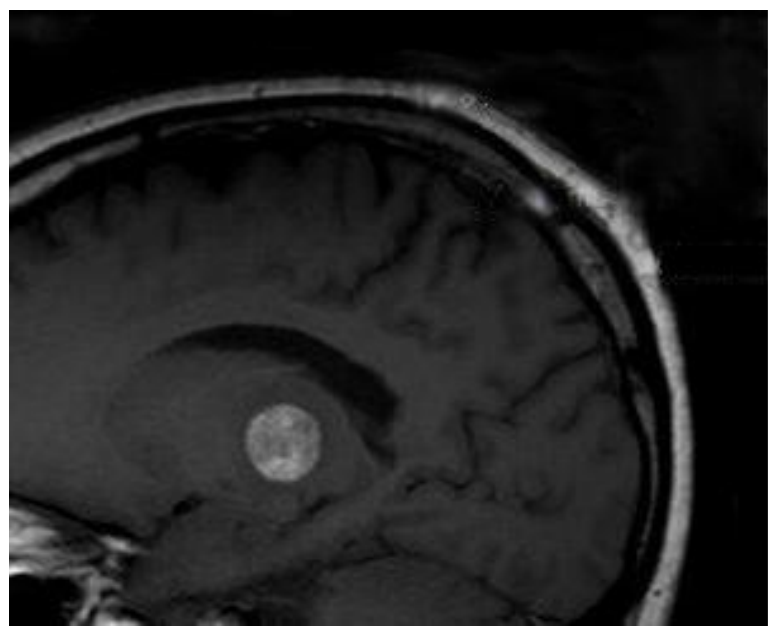

Fig -3 : Original Brain Tumor Image

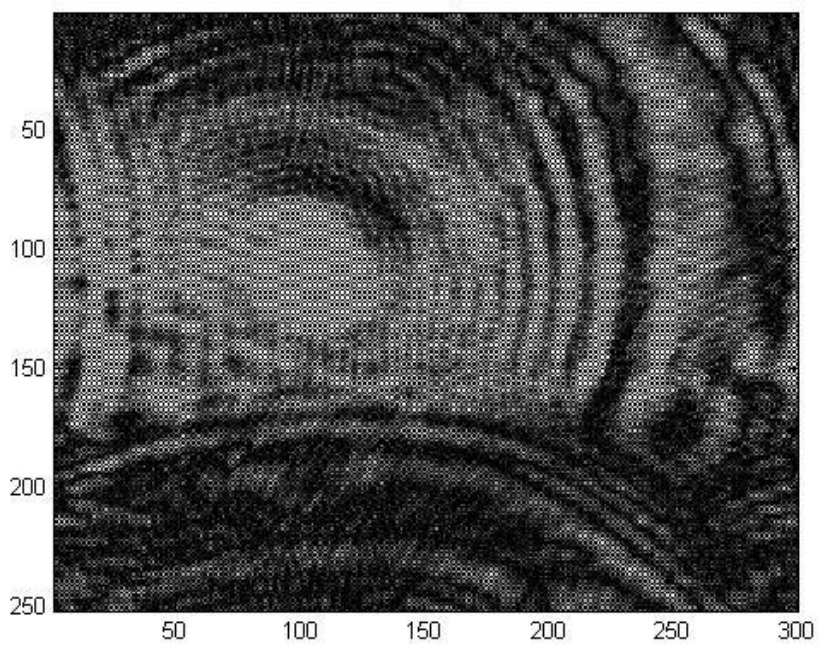

Fig -4 : Sine Hologram of Brain Tumor Image 


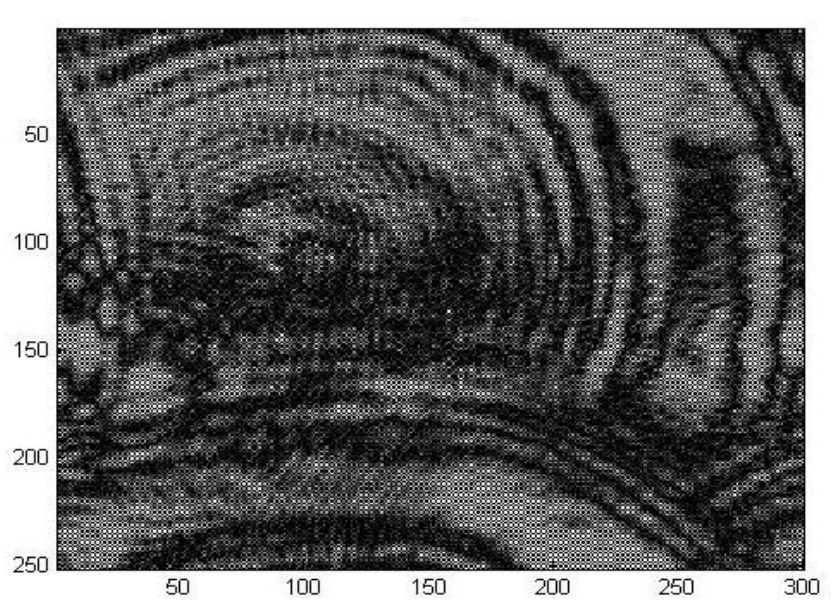

Fig -5 : Cosine Hologram of Brain Tumor Image

\section{CONCLUSIONS}

In this paper we have presented an optical scanning set up which will produce sine and cosine hologram of any biomedical image. The simulation results of construction of sine and cosine hologram are observed by using MATLAB software. The sine and cosine holograms of brain tumor image generated in this technique can be used as input for the reconstruction stage of optical set up which will visualize more information after reconstruction. So, further detection of tumor cells will become easier in real time. The sharpness, resolution and high processing speed increases the feasibility of this technique.

\section{REFERENCES}

[1]. Xin Zhang and Edmund Y. Lam "Edge Detection Of Three Dimensional Objects by Manipulating Pupil Functions in an Optical Scanning Holography System" Proceedings of 2010 IEEE $17^{\text {th }}$ International Conference on Image Processing,September 26-29,2010,Hong Kong.

[2]. Xin Zhang and Edmund Y. Lam "Sectional Image Reconstruction in Optical Scanning Holography using Compressed Sensing" Proceedings of 2010 IEEE $17^{\text {th }}$ International Conference on Image.

[3]. Ting-Chung Poon "On the fundamentals of optical scanning holography” Am. J. Phys.76(8),August 2008.

[4]. Ting-Chung Poon, Ming Hsien Wu, Kazunori Shinoda and Yoshiji Suzuki "Optical Scanning Holography" Proceedings of the IEEE, Vol.84,No.5,May1996.

[5]. Baoqun Zhao,Aili Qin, Huaying Wang, “Acquisition Of Three Dimensional Information of Minute Object by Digital Holography" 978-1-4244-2723-9/09 copyright 2009 IEEE.

[6]. W. H. Carter "Three-dimensional remote sensing by optical scanning holography: comment" Appl. Opt. 41, 5668-5671 (2002).P. Sun and J.-H. Xie, "Method for reduction of background artifacts of images in scanning holography with a Fresnel-zone-plate coded aperture" Appl. Opt. 43, 4214-4218 (2004).

[7]. J. Rosen, G. Indebetouw, and G. Brooker, "Homodyne scanning holography" Opt. Exp. 14, 4280-4285 (2006).

[8]. Ting-Chung Poon, “Optical Scanning Holography - A Review of Recent Progress" Journal of the Optical Society of Korea Vol. 13, No. 4, December 2009.

[10]. Xin Zhang and Edmund Y. Lam, “ Edge-preserving Sectional image reconstruction in optical scanning holography" J. Opt. Soc. Am. A/Vol. 27, No. 7/July 2010 .

\section{BIOGRAPHIES}

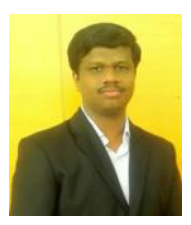

Mr. Vinay Y. Jadhav is pursuing M.Tech in VLSI Design from VIT University, Tamilnadu, India. He has completed B.Tech in Electronics and Telecommunication from RMCET, Devrukh affiliated to University of Mumbai, Maharashtra. His area of research includes Optical communication, Image processing, MEMS and Digital electronics. $\mathrm{He}$ is well versed with MATLAB and IntelliSuite ${ }^{\mathrm{TM}}$.

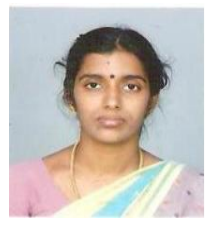

Dr.A. BRINTHA THERESE is a Professor in the School of Electronics and Communication Engineering, VIT University, Tamilnadu, India. She has an experience of 16 years, teaching in reputed engineering colleges in the capacity of the Head of the Department. She is an expert in optical image processing and working with MATLab for the past 12 years. She has organized many symposia and has presented a number of guest lecturers all over Tamilnadu, India. She has guided a number of B.Tech and M.Tech projects. She is a Life Member of the Indian Society for Technical Education. She has published many papers in Optical image processing and Optical Networking. 